\title{
Photic facilitation of tonal signal detection in a forced-choice situation'
}

MILLIAM H. WATKINS ${ }^{2}$

DECISION SCIENCES LABORATORY, L. G. HANSCOM FIELD

As Ss performed a tone detection task, background noise was reduced at possible signal presentation times. When a light stimulus intensified at those times, detectability of the tonal signal was higher than with a "dimming," or a constant light. The magnitude of intersensory facilitation was less than for experiments without acoustic time specification reported earlier.

Experiments in forced-choice tonal signal detection have been reported where light intensity variations were presented in coincidence with observation intervals (OIs) (Watkins, in press). In some of those experiments, all OIs were specified by increased amplitude of background noise. In others, a steady level was maintained. Light intensifications concurrent with OIs did not alter signal detectabilities in the former cases. but in the steady-noise experiments, intensificatione were accompanied by higher detection scores than were constant light and reciprocal (i.e., OI-diminishing) conditions.

The present experiment introduced noise amplitude reductions with OIs, to provide time specification, whlle OI-coincident light intensifications and diminutions were presented as auxiliary stimuli. A control conditionconstant light state-was also included.

The rationale which engendered this experiment was the following. If only the temporal information gained by $S$ through the task modality (audition) had been at the root of the observed difference between photic facilitation effects in the signal-embedded-in-noisebursts experiments as compared with steady background experiments, then inversion of the noise amplitude aspect of the task situation could be expected to yield data corresponding to the bursts-background results. This reasoning was, of course, predicated upon an assumption of equal time-denoting value, to practiced Ss, of positive and negative stimulus changes, equal in physical magnitude.

Method 3

Eight adult Ss were tested on a tonal signal detection task, in four-alternative, forced-choice trials, as shown in Fig. 1. $\mathrm{S}$ was seated within an anechoic chamber, with distance from head to light source controlled. Ss were given preliminary training for familiarization with the detection and response procedures. All light conditions were represented about equally in training of each $\mathrm{S}$.

A run consisted of 48 successive trials (or rarely, a few less). S began each run by operating a switch which activated the automatic stimulus presentation and data collection equipment. Subject to certain distribution restrictions, all runs had the signals occurring randomly and equally among the OIs. For any $\mathrm{S}$, a run sequence was used only once. Trials were separated by a brief illumination of a 5/8 in. "ready" lamp located above the stimulus patch. $S$ pressed one of four voting keys within each trial and learned his score at the end of the run.

Throughout the run, S was exposed, by earphones, to white noise filtered to the range $100-6800 \mathrm{cps}$. Noise amplitude was maintained at $60 \mathrm{~dB}$ overall sound Pressure Level except during each OI, when a $40 \mathrm{~dB}$ level was substituted. One of the four ( $.20 \mathrm{sec}$. duration) OIs of each trial contained a $1,011 \mathrm{cps}$ binaural signal of moderate detectability.

There were three light stimulus conditions. For condition "SB" (i.e., "steady, bright") the 1-1/2 in. circular light stimulus patch remained at its higher intensity state. In conditions "INT" and "DIM," the patch respectively intensified or diminished with all OIs by a luminance factor of about 200. Ss were instructed to watch the light patch throughout all runs. Data were collected by sets, consisting of two runs under each condition, randomly ordered.

\section{Results}

Mean percentages correct for five experimental sets are shown in Table 1 . Analyses of variance indicated that condition INT was superior to DIM $(F=8.18$, $\mathrm{df}=1 / 7, \mathrm{p}<.05)$ and to SB $(F=6.93$, df $=1 / 7, \mathrm{p}<.05)$. DIM and SB were not significantly different $(F=.94$, $\mathrm{df}=1 / 7$ ).

\section{Discussion}

Averages for tone detection in preceding experiments with background noise steady were of the order of 12

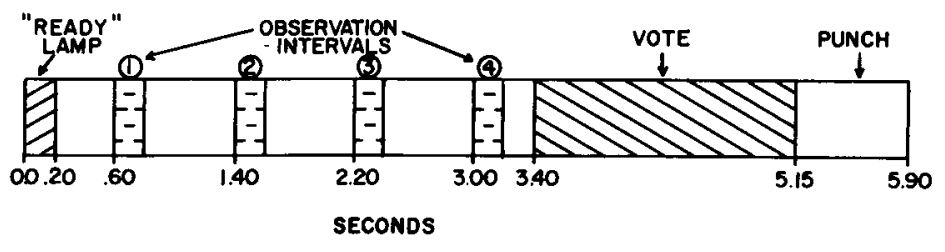

Fig. 1. Component periods of one trial, represented along a time axis. 
Table 1. Mean Percentage of Responses Correct

\begin{tabular}{llllllllllll} 
Cond. & D. E. J. O. & A. T. J. S. & A. D. A. F. A. B. D. B. Avg. \\
\hline INT & 50.1 & 75.3 & 80.7 & 63.9 & 54.5 & 64.1 & 36.0 & 61.7 & 60.8 \\
DIM & 42.1 & 70.9 & 78.0 & 63.9 & 52.9 & 57.3 & 34.0 & 62.3 & 57.7 \\
SB & 35.7 & 71.1 & 75.6 & 52.2 & 49.9 & 64.8 & 35.5 & 61.1 & 55.7 \\
\hline
\end{tabular}

points higher (percentage of responses correct) with light intensifications compared to light diminutions, each occurring in coincidence with OIs (Watkins, in press). In the present experiment the corresponding difference averaged just over three points.

It appears that two forms of prominent intra-sensory accompaniment to OIs have shown effects similar in direction (but possibly different in magnitude) upon photic facilitation in these forced-choice situations. A noise-bursts-at-OIs background essentially obliterated light condition differences in the earlier group of experiments cited. The present experiment revealed a small but still significant advantage in favor of positive photic stimuli.

An hypothesis classifying all light condition-dependent tone detection differences observed as functions of the utility of the various light conditions for "helping $S$ know when to listen" has been damaged by this experiment. That questioning of the Ss elicited no verbal reports of doubts about possible signal times may also be relevant. Despite this evidence, one can, certainly, maintain that all the light stimuli are more or less effective in the specification to $S$ of critical observation times. It is difficult to understand, however, why auditory perceptual mechanisms should rely upon visual information for temporal data, in the presence of a dependable and conspicuous auditory time cue, in the form of a $20 \mathrm{~dB}$ noise level drop at times of possible tonal signals.
A substitute, or at least supplementary, foundation for the superiority of INT over DIM and SB detection averages is believed to exist among mechanisms more subtle than those implied by the hypothesis discussed above.

An indication of divergence is noted, with regard to data recently obtained via a threshold tracking procedure by Sheridan, Cimbalo, Sills, \& Alluisi (1966) as compared to findings obtained on forced-choice tasks by the author. While the technique of Sheridan et al revealed no sensory interaction effect for a $1 \mathrm{kc}$ tonal signal, my procedures have shown some photic-facilitation-of-hearing effects at a neighboring frequency $(1,011$ cps).

\section{References}

Sheridan, J. A., Cimbalo, R. S., Sills, J. A., \& Alluisi, E. A. Effects of darkness, constant illumination, and synchronized photic stimulation on auditory sensitivity to pulsed tones. Psychon. Sci., 1966, 5, 311-312.

Watkins, W. H. Effect of intensity changes in auxiliary stimuli on auditory and visual signal detection. To appear in Proceedings of the XVIII International Congress of Psychology, Moscow 1966.

Watkins, W. H., Nickerson, R. S., \& Schjelderup, J. R. An automated facility for forced-choice signal detection experimentation. USAF ESD TDR 64-383, 1964.

\section{Notes}

1. This is ESD-TR-66-521 of the AF Electronic Systems Division, Air Force Systems Command. This research was performed at the Decision Sciences Laboratory as part of Project 7682, Man Computer Information Processing. Reproduction in whole or in part is permitted for any purpose of the U. S. Government.

2. Major, USAF. Address: ESD (ESVH, Stop 36), L. G. Hanscom Field, Bedford, Mass., 01730.

3. More detailed description of the facility apparatus, and procedures can be found in Watkins, Nickerson and Schjelderup (1964), and by request to author. 